A. Pramesh Rao, G. Swarup and Gopal-Krishna, eds.

\title{
Low Frequency Carbon Recombination Lines
}

\author{
A. A. Konovalenko \\ Institute of Radio Astronomy, National Academy of Sciences of \\ Ukraine, 4 Chervonopraporna St., 61002, Kharkov, Ukraine
}

\begin{abstract}
Low frequency carbon recombination lines with wavelengths up to twenty five meters became important means of low density interstellar plasma diagnostic. Over the past twenty years impressive amount of information was obtained for Cassiopeia A and other galactic directions.
\end{abstract}

\section{Introduction}

Until the end of 1970's investigations of the astrophysical phenomenon of radio recombination lines (RRL) were a privilege of high frequency radio astronomy $(\nu>1 \mathrm{GHz})$. The lowest frequency hydrogen line is $\mathrm{H} 352 \alpha$ feature at 150 $\mathrm{MHz}$, detected in hot gas towards the Galactic Center (Anantharamaiah, Payne, $\&$ Bhatacharya 1990). In spite of this, the importance of low frequency RRL investigation became clear many years ago. According to theoretical estimations (Shaver 1975), rather intense (due to stimulated emission) low frequency $(\nu=$ $100-300 \mathrm{MHz}$ ) hydrogen lines are expected to be formed in diffused and cold $\left(T_{e} \sim 100 \mathrm{~K}, N_{e} \sim 0.03 \mathrm{~cm}^{-3}\right)$ interstellar components, which are heated and ionized by cosmic rays and X-rays. Surprisingly, these phenomena are still not detected, and the estimates of the hydrogen ionization rate is $\xi_{H}<10^{-17} \mathrm{~s}^{-1}$ (hydrogen is practically neutral in these components).

Detection of spectral features in absorption at extremely low frequencies $(\nu<30 \mathrm{MHz}$ ) (Konovalenko \& Sodin 1980) with the radio telescope UTR2 (they turned out to be RRL of strongly excited carbon atoms with $n \sim 630$ (Blake, Crutcher, \& Watson 1980; Konovalenko \& Sodin 1981) opened new ways of studying the low density interstellar plasma using low and very low frequency radio spectroscopy methods.

\section{Low Density Interstellar Plasma and the Role of Carbon Atoms}

Physical conditions and processes in the interstellar medium (ISM) are dramatically varied, especially for interstellar gas (Spitzer 1978). It is important to note that practically all gas is ionized at least at a low rate. The most part of the gas (completely or partially ionized) outside of HII regions has electron density $N_{e}<1 \mathrm{~cm}^{-3}$. Its temperature could be both high $\left(T_{e}>10^{3} \mathrm{~K}\right)$ and low $\left(T_{e}<100 \mathrm{~K}\right)$. This gas plays a significant role in energetic, dynamic, and evolution of the galactic matter. UV photons with $\lambda>912 \AA$ escape from HII regions and could ionize elements having ionization potential less than HI. Other 


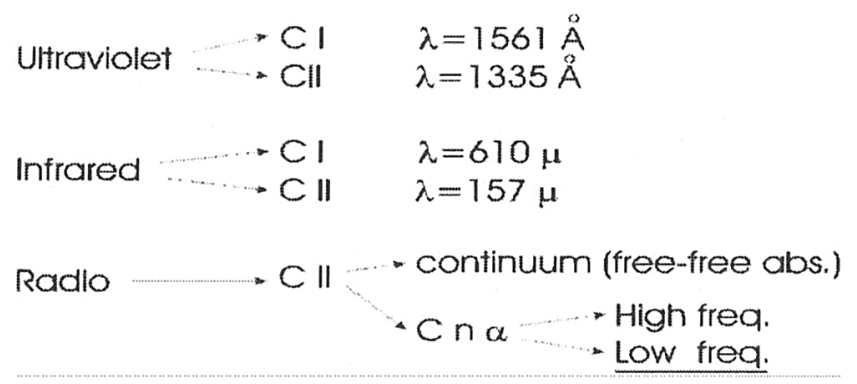

Figure 1. Astrophysical methods of interstellar carbon observations.

sources of low density plasma (both cold and hot components) ionization and heating could exist also.

The role of carbon as one of the main source of electrons and ions in the cold ISM should be stressed. The reasons for the significance of carbon atom in the ISM are the following: carbon is the most abundant element having ionization potential less than that of hydrogen $\left(C / H=3.7 \times 10^{-4}, E_{c}=11.2\right.$ $\left.\mathrm{eV}, E_{H}=13.6 \mathrm{eV}\right)$; it is almost completely ionized in diffuse interstellar gas; it is the main element that determines cooling and thermostatic processes in HI clouds due to fine structure transition ${ }^{2} P_{3 / 2}-{ }^{2} P_{1 / 2}$ with $\lambda=157 \mu$; most of the interstellar molecules contain carbon atoms; carbon plays a significant role in gas-phase chemical reactions and effectively reflects various physical processes in the ISM.

Neutral and ionized carbon could be observed with several astrophysical methods. Some of them are presented in Fig. 1. The radio astronomy approach, particularly carbon RRL observations, might be the most promising one.

The first detection of carbon RRL was made at high frequencies (Palmer et al. 1967). After that they were observed in the directions of good many HII regions simultaneously with corresponding $\mathrm{Hn} \alpha$ lines. As follows from atomic physics, the mechanism of carbon line formation is similar to that of hydrogen lines: there is only an isotopic shift.

Now it has been established that carbon lines in the directions of HII regions arise in cold $\left(T_{e} \sim 100 \mathrm{~K}\right)$ gas, lying over HII region periphery. Based on the above statements, some simple models of objects, where carbon RRL could arise, are presented in Fig. 2. It should be stressed that for the objects of type 2 and 3 (having no Hn $\alpha$ lines) carbon RRL at high frequencies were detected only in several selected regions (Brown 1980; Crutcher 1977). But, fortunately, absolutely new opportunities in RRL studies have been opened up by radio spectroscopy at low frequencies.

\section{Low Frequency RRL in the Direction of Cassiopeia A}

Carbon RRL detected at decameter waves towards Cas A have the following particular characteristics: they are the first RRL observed in absorption; line broadening is dramatically strong and surpasses all observed in astrophysics previously; association of these lines with rather cold gas in diffuse CII regions 


\section{H II regions 2.Dark dust clouds 3.Diffuse clouds}

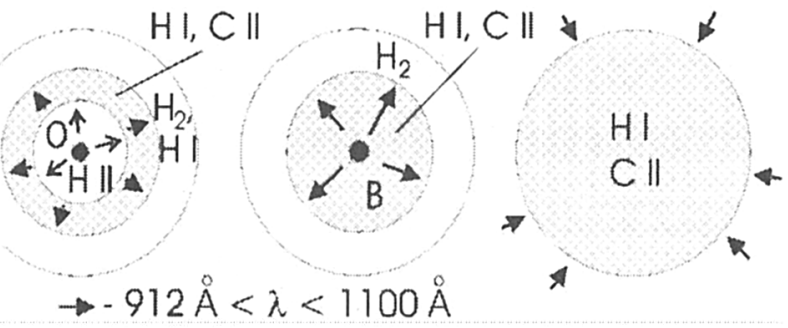

Figure 2. Possible interstellar medium objects with ionized carbon.

not connected to HII ones is a reasonable answer to question where they are arisen because $\mathrm{Hn} \alpha$ lines are not detected in the corresponding directions.

Using Shaver's theory, it was suggested that the lines should turn to emission at higher frequencies (Konvalenko \& Sodin 1981). Such features were first detected at $\nu>200 \mathrm{MHz}$ (Payne, Anantharamaiah, \& Erickson 1989). Absorption-emission turnover reliably confirms the existence of stimulated emission in partially ionized low density gas lying against a strong continuum radio source. Substantial variation of line width and intensity, including change of polarity (such a turnover in not observed at high frequencies), gives excellent opportunity for diagnostics of the rarefied interstellar plasma. So it is not surprising that $\mathrm{Cn} \alpha$ lines are intensively studied at many radio telescopes, including UTR-2 and RT-70 (Ukraine), GEE-TEE and Ooty (India), DKR-1000 and RT22 (Russia), Green Bank, the VLA, and Arecibo (USA), Effelsberg (Germany), Parkes (Australia) (Anantharamaiah, Erickson, \& Radhakrishnan 1985; Kantharia, Anantharamaiah, \& Payne 1998 and references therein).

Cas A is the strongest radio source, against which RRL are observed. In this case antenna temperature sufficiently exceed background one even for small antennas. So the same region of the ISM is investigated in all radio observations independent of the beam size (Fig. 3). Thus, this source provides an opportunity of the most accurate determination of line and medium characteristics. It would not be any exaggeration to say that Cas A is the "corner-stone" of low frequency radio spectroscopy (Konovalenko 1990).

The Cn $\alpha$ lines along Cas A line of sight have been observed in very wide frequency range from $15 \mathrm{MHz}(n \sim 800)$ up to $1400 \mathrm{MHz}(n \sim 160)$. The number of detected features comes to several tens, which is unprecedented compared to other objects studied with RRL.

Quite recently the lowest frequency spectral lines $(n \sim 812)$ were detected near $12 \mathrm{MHz}$ with radio telescope UTR-2. Atoms with the highest principal quantum numbers of about 860 were observed using $\beta$-lines near $20 \mathrm{MHz}$ at the same instrument (see Fig. 4).

As follows from theoretical studies, low frequency line parameters (firstly, integral line intensity and pressure broadening) are strongly dependent on temperature $T_{e}$, electron density $N_{e}$, path length $S$, and principal quantum number $n$. But the decisive factors are departure coefficients $b_{n}$ and $\beta_{n}$, which determines a character of lines behavior, including their polarity. In 1980 it was shown that 


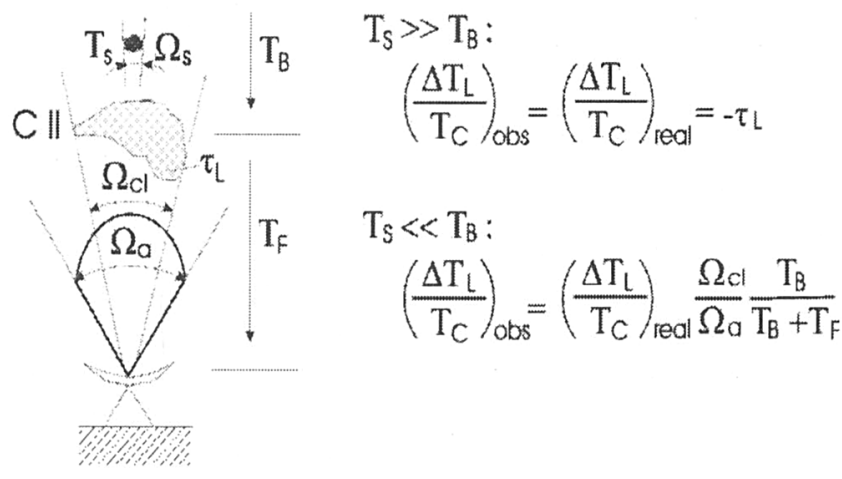

Figure 3. Diagram of LF Cn $\alpha$ lines observations.

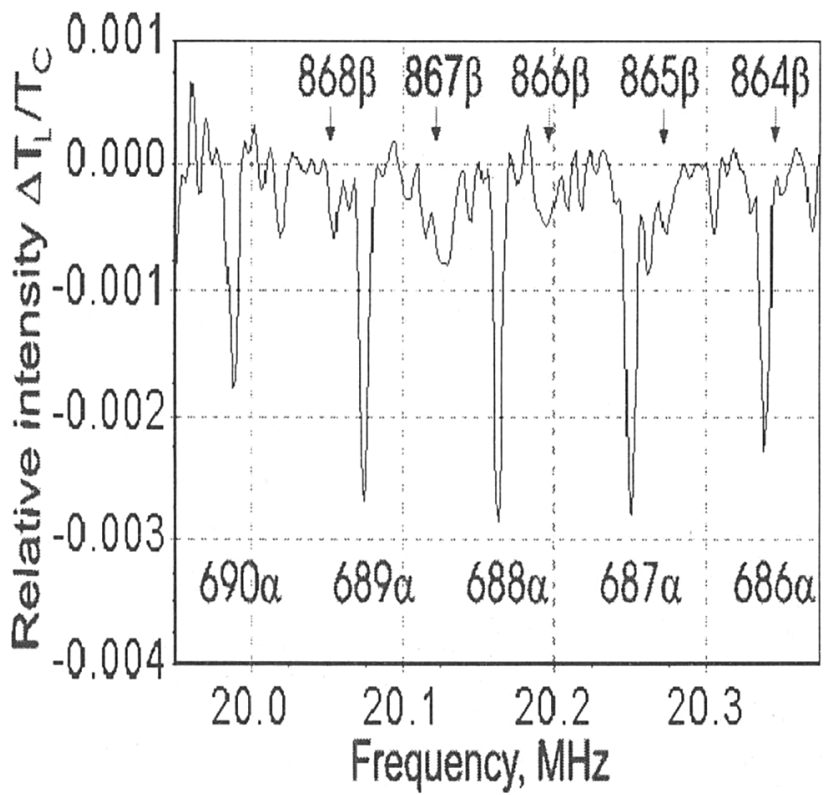

Figure 4. A series of carbon RRL near $20 \mathrm{MHz}$. 
a mechanism of dielecronic-like recombination, existing due to fine structure transition ${ }^{2} P_{3 / 2}-{ }^{2} P_{1 / 2}(\Delta T=92 \mathrm{~K})$, can considerably modify departure coefficients of carbon ion states (Watson, Western, \& Christensen 1980). Detailed calculations (Walmsley \& Watson 1982) showed that in the case of $T_{e} \sim 100 \mathrm{~K}$ $b_{n} \beta_{n}$ can reach the values of $10-100$. One of other possible mechanism of $b_{n} \beta_{n}$ modification is the underpopulation of high atomic levels $\left(b_{n} \rightarrow 0\right.$ with $\left.n \rightarrow \infty\right)$ (Gulyaev \& Nefedov 1989; Payne, Anantharamaiah, \& Erickson 1994). It may be noted that classical hydrogenic-like recombination $\left(b_{n} \rightarrow 1\right.$ with $\left.n \rightarrow \infty\right)$ gives the value of $b_{n} \beta_{n}$ of about 1 for high $n$ (Shaver 1975). Exotic mechanism of high temperature $\left(T_{e}>10^{4} \mathrm{~K}\right)$ dielectronic recombination can provide $b_{n} \beta_{n} \sim 10^{3}$ for heavy elements (Shaver 1976). The area where $d l_{n} b_{n} / d_{n}$ is negative is of particular interest: this leads to strong amplification of absorption lines at the lowest frequencies that yields the unique opportunity of plasma diagnostics for a great number of the interstellar objects even when electron density is less than $0.1 \mathrm{~cm}^{-3}$.

In order to build a comprehensive physical model of the medium, it is necessary to fit the calculation data with wide band experimental results. Moreover, other astrophysical information on the ISM properties, including primarily data of $\mathrm{HI}$ and molecular lines, interstellar pressure, and thermal equilibrium, must be taken into account (Payne, Anantharamaiah, \& Erickson 1994).

Two types of models for the medium were proposed:

1. Cold gas model with $T_{e} \sim 20 \mathrm{~K}, N_{e} \sim 0.3 \mathrm{~cm}^{-3}$, and hydrogenic-like level's population; CII regions are associated with cold, clumped, and mainly molecular gas (Ershov et al. 1984; Sorchenko \& Walmsley 1994).

2. Warm gas model with $T_{e} \sim 100 \mathrm{~K}, N_{e} \sim 0.05 \mathrm{~cm}^{-3}$, and dielectronic-like recombination; regions of line formation are associated with diffuse HI clouds and are themselves diffuse (Payne, Anantharamaiah, \& Erickson 1989; Walmsley \& Watson 1982; Konovalenko 1984a).

Base on available information it can be concluded that the warm gas model is in better agreement with experimental data. The VLA mapping of C274 $\alpha$ line distribution confirmes this (Kantharia, Anantharamaiah, \& Payne 1998). Kantharia, Anantharamaiah, \& Payne (1998) proposed an additional broadening effect, caused by non-thermal radio emission from Cas A. The best-fit warm gas model parameters are $T_{e}=75 \mathrm{~K}, N_{e}=0.02 \mathrm{~cm}^{-3}$, and $M E=0.011 \mathrm{~cm}^{-6}$ pc. For this kind of models a $n_{H} T_{e}$ factor of about $10^{4} \mathrm{Kcm}^{-3}$ becomes reasonable. It should be emphasized that line-of-sight size of the region is near $30 \mathrm{pc}$ that is sufficiently bigger that linear size of Cas $\mathrm{A}(\sim 7 \mathrm{pc})$.

\section{Observations of Low Frequency Carbon RRL in the Galaxy}

Successful observations and fruitful analyses of $\operatorname{Cn} \alpha$ towards Cas A motivated the search for such features in the directions to other objects. Galactic background brightness temperature $(\sim 30,000 \mathrm{~K}$ at $25 \mathrm{MHz})$ will always exceed electron temperature of an investigated region throughout the Galaxy. However, values of observed relative line intensities (as it is seen in Fig. 3) are less than real ones in the first turn because of telescope beam dilution as well as foreground emission (especially when objects are distant). The integration time needed for reliable detection or estimation of upper limits to line parameter 
can reach hundred hours. In spite of this, the promising perspectives of these investigations justify these efforts.

A search for $\mathrm{Cn} \alpha$ lines near $25 \mathrm{MHz}(n \sim 640)$ in various galactic objects has been carried out over the past two decades with the world largest decameter wave array UTR-2 and 128 channel digital correlater. In the first turn it is very interesting to carry out investigations in the directions of regions near the galactic plane, towards maximums of HI column density $\left(N_{H}>10^{20} \mathrm{~cm}^{-2}\right)$, as well as towards HII regions, SNR, and dark dust and giant molecular clouds. Positive results were obtained for many objects. Among them are the following: NGC 2024; $l=75^{\circ}, b=0^{\circ}$; S140; DR-21; L1407; $\rho$ Oph; M16; Per OB2; $l=35^{\circ}$, $b=0^{\circ} ; \alpha=17^{h}, \delta=70^{\circ}$ (Golynkin \& Konovalenko 1991a; 1991b).

Investigations of regions along the line-of-sight to areas near the Galactic Center have been carried out systematically with radio telescopes in Parkes at $75 \mathrm{MHz}$ (Erickson, McConnell, \& Anantharamaiah 1995), Garibidanur at 34.5 $\mathrm{MHz}$ (Kantharia \& Anantharamaiah 1999), and Ooty at $328 \mathrm{MHz}$ (Kantharia \& Anantharamaiah 1999; Roshi \& Anantharamaiah 1997). Thirty positions with galactic longitude lying in the interval of $l=145^{\circ}-342^{\circ}$ (steps was $5^{\circ}-15^{\circ}$ ) were observed at $34.5 \mathrm{MHz}$. Among other positive results are measurements in the directions of $l=63^{\circ}, l=75^{\circ}$, and DR-21 $\left(l \approx 82^{\circ}\right)$. The data of the last two observations are in good agreement with UTR-2 data.

It is expected that line width does not depend upon $n$ for the most of the directions in frequency range $25-328 \mathrm{MHz}\left(\Delta \nu_{L}=20-50 \mathrm{MHz}\right)$ (Kantharia \& Anantharamaiah 1999). However, for the Cas A direction $\Delta \nu_{L}$ changes from $5 \mathrm{~km} / \mathrm{s}$ to $70 \mathrm{~km} / \mathrm{s}$. Possibly, the line width is determined only by systematic and turbulent gas movement. The upper limit of electron density is $N_{e}<0.3$ $\mathrm{cm}^{-3}$.

Using measurements at three frequencies for the inner part of the Galaxy and angular size estimation $\left(2^{\circ}-4^{\circ}\right)$, the most feasible values for the properties of the medium were (Kantharia \& Anantharamaiah 1999): $T_{e}=40-100 \mathrm{~K}, N_{e}$ $=0.003-0.01$, and path length more than several parsecs. Thus, low frequency $\mathrm{Cn} \alpha$ lines arise mainly in diffuse rather warm CII regions associated with HI gas where conditions for dielectronic-like process are existed.

\section{Future Perspectives of Low Frequency RRL Investigation}

During the last years the interest for low frequency radio astronomy has grown considerably. This relates to new possibilities opened by low frequency carbon RRL investigations.

The radio telescope UTR-2 remains the most effective instrument at frequencies less than $30 \mathrm{MHz}$. Among its principal features are the biggest effective area, high directivity, broad band, electronic beam steering, and multi-beam working regimes. For spectral investigations there is no sensitivity limitation due to confusion effect. Recent upgrade of preamplifier system has yielded uninterrupted frequency coverage and good interference immunity, which are extremely important for spectral line observation (Abranin et al. 1997).

Many radio telescopes at frequencies greater than $30 \mathrm{MHz}$ have undergone substantial upgrades. New sensitive and efficient radio telescopes like the GMRT, which is very suitable for RRL investigations at $50-1400 \mathrm{MHz}$, are 
being built. Without any doubt, the development of low frequency carbon RRL radio spectroscopy should be included among scientific motivations of the creation of new generations of giant low frequency arrays.

The progress of modern digital electronics and computer techniques provides effective ways of development of wide-band (up to several tens of $\mathrm{MHz}$ ) and multi-channel (up to many thousands) digital correlometers and spectral processors. Such a new correlometer with maximum sampling rate of $60 \mathrm{MHz}$ and 4096 channels was installed at UTR-2 observatory (Konovalenko et al. 1999).

The considerable amount of data obtained for the Cas A direction does not exclude the further developments and studies. There are promising ways of improving measurement accuracy and Voight profile fitting improvement, especially at the lowest frequencies.

Systematic search and study of $\operatorname{Cn} \alpha$ lines in the galactic plane direction at very low $(\nu<30 \mathrm{MHz})$ and low $(\nu \sim 330 \mathrm{MHz})$ frequencies are very important. Available results and estimates show that above discussed features could be detected in a good many galactic directions using existing radio telescopes and those under construction, employing new and more sophisticated experimental techniques. The relative line intensity to be measured will be of about $10^{-3}$ $10^{-4}$. This sensitivity level provides a possibility to obtain valuable information about physical parameters and processes in the ISM.

\section{Conclusion}

Carbon RRL at low frequencies open new opportunities for rarified interstellar plasma diagnostics and Rydberg atom physics studies. While they are now not exotic phenomena, their properties and the conditions in the regions of their formation remain mysterious to a great extent and are still very interesting for investigation. In spite of numerous difficulties of detection and interpretation, low frequency carbon RRL yield unique information not available with other astrophysical methods.

Acknowledgments. The author is thankful to G. Swarup and IAU 199 Symposium program committee who proposed to make this review and provided crucial support at URSI committee for travel grant allocation. This paper was written during the author stay at Raman Research Institute, Bangalore, India. Many thanks to K. R. Anantharamaiah, R. Nitjananda, V. Radhakrishnan, and all Raman Research Institute staff for invitation and hospitality. The author gives thanks to K. R. Anantharamaiah for fruitful discussion and critical reading of the manuscript as well as to UTR-2 colleagues for taking part in the investigations. This activity is supported in part by grants INTAS 96-0183, 97-1964, and INTAS-CNES 97-1450.

\section{References}

Abranin, E. P., Bruk, Yu. M., Zakharenko, V. V., \& Konovalenko, A. A. 1997, Radiophysics \& Radioastronomy, 2, 95

Anantharamaiah, K. R., Erickson, W. C., \& Radhakrishnan, V. 1985, Nature, 315, 647 
Anantharamaiah, K. R., Payne, M. E., \& Bhatacharya, D. 1990, in Radio Recombination Lines: 25 Years of Investigations, eds. M. A. Gordon, R. L. Sorochenko (Dordrecht: Kluwer), 259

Blake, D. H., Crutcher, R. M., \& Watson, W. D. 1980, Nature, 287, 707

Brown, R. L. 1980, in Radio Recombination Lines, ed. P. A. Shaver (Dordrecht: Reidel), 127

Crutcher, R. M. 1997, ApJ, 217, L109

Erickson, W. C., McConnel, D., \& Anantharamaiah, K. R. 1995, ApJ, 454, 125

Ershov, A. A., Ilyasov, Yu. P., Lekht, E. E., Smirnov, G. T., Solodkov, V. T., \& Sorochenko, R. L. 1984, Soviet. Astron. Lett., 10, 348

Golynkin, A. A., \& Konovalenko, A. A. 1991a, Sov. Astron. Lett., 17, 16

Golynkin, A. A., \& Konovalenko, A. A. 1991b, Sov. Astron. Lett., 17, 23

Gulyaev, S. A., \& Nefedov, S. A. 1989, Astron. Nachr., 310, 403

Kantharia, N. G., Anantharamaiah, K. R., \& Payne, H. E. 1998, ApJ, 506, 758

Kantharia, N. G., \& Anantharamaiah, K. R. 1999 (in preparation)

Konovalenko, A. A. 1984a, Sov. Astron. Lett., 10, 353

Konovalenko, A. A. 1984b, Sov. Astron. Lett., 10, 912

Konovalenko, A. A. 1990, in Radio Recombination Lines: 25 years of investigations, eds. M. A. Gordon, R. L. Sorochenko (Dordrecht: Kluwer), 175

Konovalenko, A. A., \& Sodin, L. G. 1980, Nature, 283, 360

Konovalenko, A. A., \& Sodin, L. G. 1981, Nature, 294, 135

Konovalenko, A. A., Stepkin, S. V., Shalunov, D. V., \& Erickson, W. C. 1999 (in preparation)

Palmer, P., Zuckerman, B., Penfield, H., Liley, A. E., \& Mezger, P. G. 1967, Nature, 215,40

Payne, H. E., Anantharamaiah, K. R., \& Erickson, W. C. 1989, ApJ, 341, 890

Payne, H. E., Anantharamaiah, K. R., \& Erickson, W. C. 1994, ApJ, 430, 690

Roshi, D. A., \& Anantharamaiah, K. R. 1997, MNRAS, 292, 63

Shaver, P. A. 1975, Pramana, 5, 1, 1

Shaver, P. A. 1976 , A\&A, 46, 127

Sorochenko, R. L., Wamsley, C. M. 1991, A\&A Trans., 1, 31

Spitzer, L. 1978, Physical Prosseses in the Interstellar Medium (New York: Wiley)

Walmsley, C. M., \& Watson, W. D. 1982, ApJ, 240, 956

Watson, W. D., Western, L. R., \& Christensen, R. B. 1980, ApJ, 240, 956 